\title{
Effect of extender oils on the stress relaxation behavior of thermoplastic vulcanizates
}

\author{
H. H. Le, Q. Zia, S. Ilisch, H. J. Radusch* \\ Martin Luther University Halle-Wittenberg, Center of Engineering Sciences, Chair Polymer Technology, \\ D-06099 Halle (Saale), Germany
}

Received 3 September 2008; accepted in revised form 23 September 2008

\begin{abstract}
The long term mechanical behavior of oil extended thermoplastic vulcanizates (TPV) based on polypropylene (PP) and acrylonitrile-butadiene rubber (NBR) has been characterized by means of stress relaxation experiments. The morphology of TPV and the phase specific oil distribution which depend on the content and type of oil as well as on the mixing regime have been characterized by means of Atomic Force Microscopy (AFM), Dynamic Mechanical Thermal Analysis (DMTA) and Differential Scanning Calorimetrie (DSC). The discussion of the stress relaxation behavior was carried out using the two-component model, which allows splitting the initial stress into two components: a thermal activated stress component and an athermal one. A master curve was created by shifting the relaxation curves vertically and horizontally towards the reference curve. The vertical shift factor $b_{T}$ is a function of the temperature dependence of the athermal stress components. It was found that the oil distribution strongly affects the athermal stress component which is related to the contribution of the structural changes, e.g. crystallinity of the PP phase and the average molecular weight between the crosslinks of the NBR phase. From the temperature dependence of the horizontal shift factor $a_{T}$ the main viscoelastic relaxation process was determined as the $\alpha$-relaxation process of the crystalline PP phase. It is not dependent on the polarity and content of the oil as well as the mixing regime.
\end{abstract}

Keywords: polymer blends and alloys, thermoplastic vulcanizates, stress relaxation, extender oil

\section{Introduction}

Thermoplastic vulcanizates (TPV) are produced by melt mixing of an elastomer with a thermoplastic component and simultaneously but selective crosslinking of the elastomer phase during melt mixing. The resulting blend comprises a thermoplastic resin filled with a high concentration of dispersed rubber particles [1-3]. In order to improve the processability, reduce the material cost and enhance the performance of the end-use products, oil often has been added into thermoplastic vulcanizates. The addition of extender oil alters the viscoelastic properties of these heterogeneous thermoplastic vulcanizates significantly. Although the viscoelastic behavior of heterogeneous thermoplastic-rubber systems was already investigated [4-7], the influence of the extender oil on the relaxation behavior of such systems was not sufficiently investigated so far.

In the present work the two-component model [8-10] was used to characterize the effect of extender oils on the stress relaxation behavior of TPV by correlating the structural features and the effect of oil content, polarity and distribution on it.

\section{Experimental}

\subsection{Materials}

NBR (Perbunan NT 3465, Bayer, Germany) with acrylonitrile content of $33 \%$ and iPP (Stamylan P14, Sabic, Germany) were used as elastomeric and

*Corresponding author, e-mail: hans-joachim.radusch@iw.uni-halle.de (C) BME-PT and GTE 
Table 1. Formulation of TPV-oil mixtures

\begin{tabular}{|c|c|c|c|c|}
\hline Sample name & TPV content [wt \%] & Oil content [wt\%] & Oil type & Oil addition \\
\hline TPV & 100 & 0 & - & - \\
\hline A-N-10 & 90 & 10 & \multirow{3}{*}{ Non-polar } & \multirow{3}{*}{ Oil added after dynamic vulcanization process } \\
\hline A-N-20 & 80 & 20 & & \\
\hline A-N-30 & 70 & 30 & & \\
\hline A-P-10 & 90 & 10 & \multirow{3}{*}{ Polar } & \multirow{3}{*}{ Oil added after dynamic vulcanization process } \\
\hline A-P-20 & 80 & 20 & & \\
\hline A-P-30 & 70 & 30 & & \\
\hline B-P-10 & 90 & 10 & \multirow{3}{*}{ Polar } & \multirow{3}{*}{$\begin{array}{l}\text { Oil premixed into rubber phase before dynamic vul } \\
\text { canization process }\end{array}$} \\
\hline B-P-20 & 80 & 20 & & \\
\hline B-P-30 & 70 & 30 & & \\
\hline
\end{tabular}

thermoplastic components, respectively. The ratio of NBR to PP was kept 65 to 35. Phenolic resin (Vulkaresen PA 510, Vianova Resins, Germany) acted as cross-linking agent. Compatibilizer used was separately prepared by mixing $75 \mathrm{wt} \%$ maleic anhydride functionalized PP (Exxelor PO 1020, Exxon, Germany) and $25 \mathrm{wt} \%$ amine terminated NBR (Hycar ATNB 1300 X16, Goodrich, Germany). A polar oil (Enerdex 65, BP/Tudalen-65, $\mathrm{H} \& \mathrm{R}$ Group, Germany) having a freezing temperature of $-37^{\circ} \mathrm{C}$ and polar bonds of $8 \mathrm{wt} \%$ and a non-polar oil (Enerpar 1927, BP/Tudalen-1927, $\mathrm{H} \& \mathrm{R}$ Group, Germany) having a freezing temperature of $-63^{\circ} \mathrm{C}$ and polar bonds of $1.2 \mathrm{wt} \%$ were used, respectively. The formulations of polymer-oil mixtures are shown in Table 1.

\subsection{Mixing regime and sample preparation}

Thermoplastic vulcanizates were prepared using a double-rotor lab mixer (Plasticoder PL-2100, Brabender, Germany) with rotor speed of $65 \mathrm{rpm}$ at a starting temperature of $180^{\circ} \mathrm{C}$. The mixing regime is given in Table 2 and Table 3 for different mix-

Table 2. Mixing regime for A-N and A-P samples

\begin{tabular}{|c|l|}
\hline Mixing time [min] & \multicolumn{1}{c|}{ Ingredients } \\
\hline 0 & PP + Compatibilizer \\
\hline 2 & NBR \\
\hline 4 & $\begin{array}{l}\text { Cross-linking agent } \\
\text { Non-polar oil (A-N samples) } \\
\text { Polar oil (A-P samples) }\end{array}$ \\
\hline 6 & Stop \\
\hline 12 & \\
\hline
\end{tabular}

Table 3. Mixing regime for B-P samples

\begin{tabular}{|c|l|}
\hline Mixing time [min] & \multicolumn{1}{|c|}{ Ingredients } \\
\hline 0 & NBR \\
\hline 2 & Polar oil \\
\hline 4 & PP + Compatibilizer \\
\hline 7 & Cross-linking agent \\
\hline 12 & Stop \\
\hline
\end{tabular}

tures. After discharge from the mixer, all samples were compression molded at a temperature of $200^{\circ} \mathrm{C}$ and pressure of 70 bar to produce sheets of $0.3 \mathrm{~mm}$ thickness for stress relaxation experiments and $1 \mathrm{~mm}$ specimens for the other test methods.

\subsection{Morphology characterization, thermal and mechanical testing}

The phase morphology was investigated using an Atomic Force Microscope (Q-Scope 250, Quesant) under intermittendent mode. The cantilever stiffness is $40 \mathrm{~N} / \mathrm{m}$ and the resonance frequency nearly $170 \mathrm{kHz}$. Samples were cryo-cutted by means of a microtome (HM 360/CM 30, Microm) at $-110^{\circ} \mathrm{C}$ with a diamond knife in order to get a smooth surface of the sample.

A heat flux calorimeter (DSC 820, Mettler Toledo) equipped with a liquid nitrogen cooling assembly was used to investigate the thermal behavior of the blends. Temperature range selected was from -80 to $180^{\circ} \mathrm{C}$ with a heating and cooling rate of $10 \mathrm{~K} / \mathrm{min}$.

Dynamic mechanical behavior was characterized using a dynamic mechanical analyzer (Qualimeter Eplexor 500 N, Gabo) in tensile mode at a frequency of $1 \mathrm{~Hz}$ at different temperatures $\left(-135^{\circ} \mathrm{C}<\right.$ $T<170^{\circ} \mathrm{C}$ ) with a heating rate of $1 \mathrm{~K} / \mathrm{min}$.

Tensile tests of all the blends were carried out according to EN ISO 527 using a universal testing machine (Zwick 1425, Zwick/Roell) at room temperature. Dumbbell-shaped specimens were used with an initial length of $20 \mathrm{~mm}$; the clamp distance was $50 \mathrm{~mm}$. A crosshead speed of $50 \mathrm{~mm} / \mathrm{min}$ was applied. Minimum five samples were tested.

For the determination of the residual strain after a hysteresis run, dumbbell-shaped specimens were used with the initial length of $20 \mathrm{~mm}$. Samples 
were strained with a crosshead speed of $50 \mathrm{~mm} / \mathrm{min}$ to an elongation of $100 \%$. For release, the same rate was used. The residual strain is a measure for the elastic behaviour.

Stress relaxation experiments were performed using a dynamic mechanical analyzer (DMTA Mark 3E, Rheometric Scientific) in tensile mode. The instrument was equipped with a temperature chamber enabling a long-term constancy of the temperature (deviation $<0.1 \mathrm{~K}$ ). Relaxation curves for all the samples were recorded at a draw ratio of $\lambda=1.5(\varepsilon=50 \%)$ within the temperature range from 30 to $120^{\circ} \mathrm{C}$ over a period of 30 minutes to 15 hours depending on test temperature and oil content. The two-component model used in the present study has been described in a previous work [4]. No steady state stress value was observed even after 15 hours. Therefore, an extrapolation method proposed by Li [11] was used to determine the athermal stress component $\sigma_{\infty}$.

\section{Results}

\subsection{Morphology characterization of oil extended TPV}

The blend morphology and the phase specific oil distribution in TPV have been characterized using the AFM images and the DMTA results presented in Figure 1 and Figure 2. Figures $1 \mathrm{a}$ and $1 \mathrm{~b}$ show the morphology of TPV filled with different oil

a)
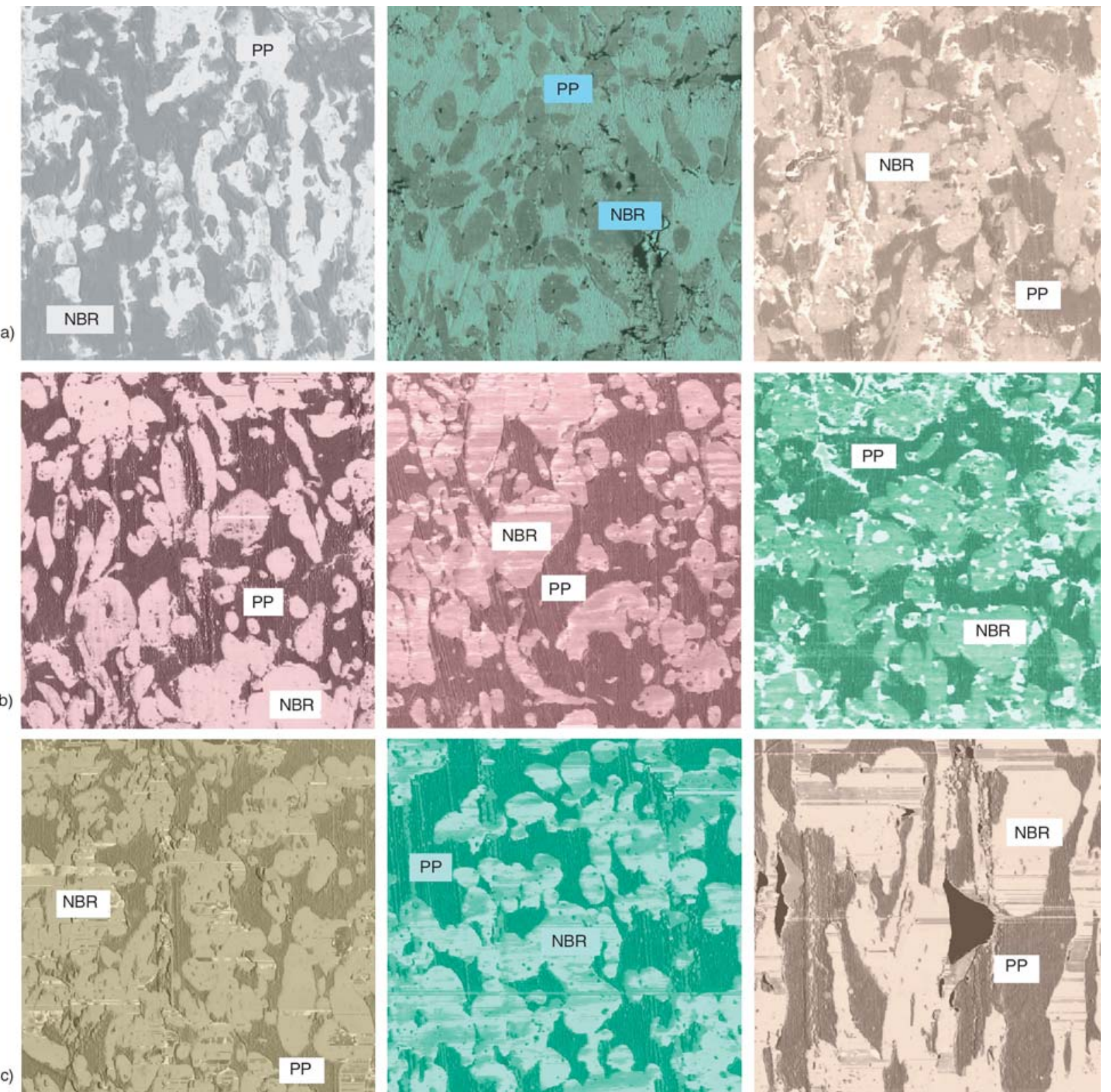

Figure 1. AFM images of different TPV: A-N-10, A-N-20 and A-N-30 (a), A-P-10, A-P-20 and A-P-30 (b), B-P-10, B-P-20 and B-P-30 (c), image size $20 \mu \mathrm{m} \times 20 \mu \mathrm{m}$ 

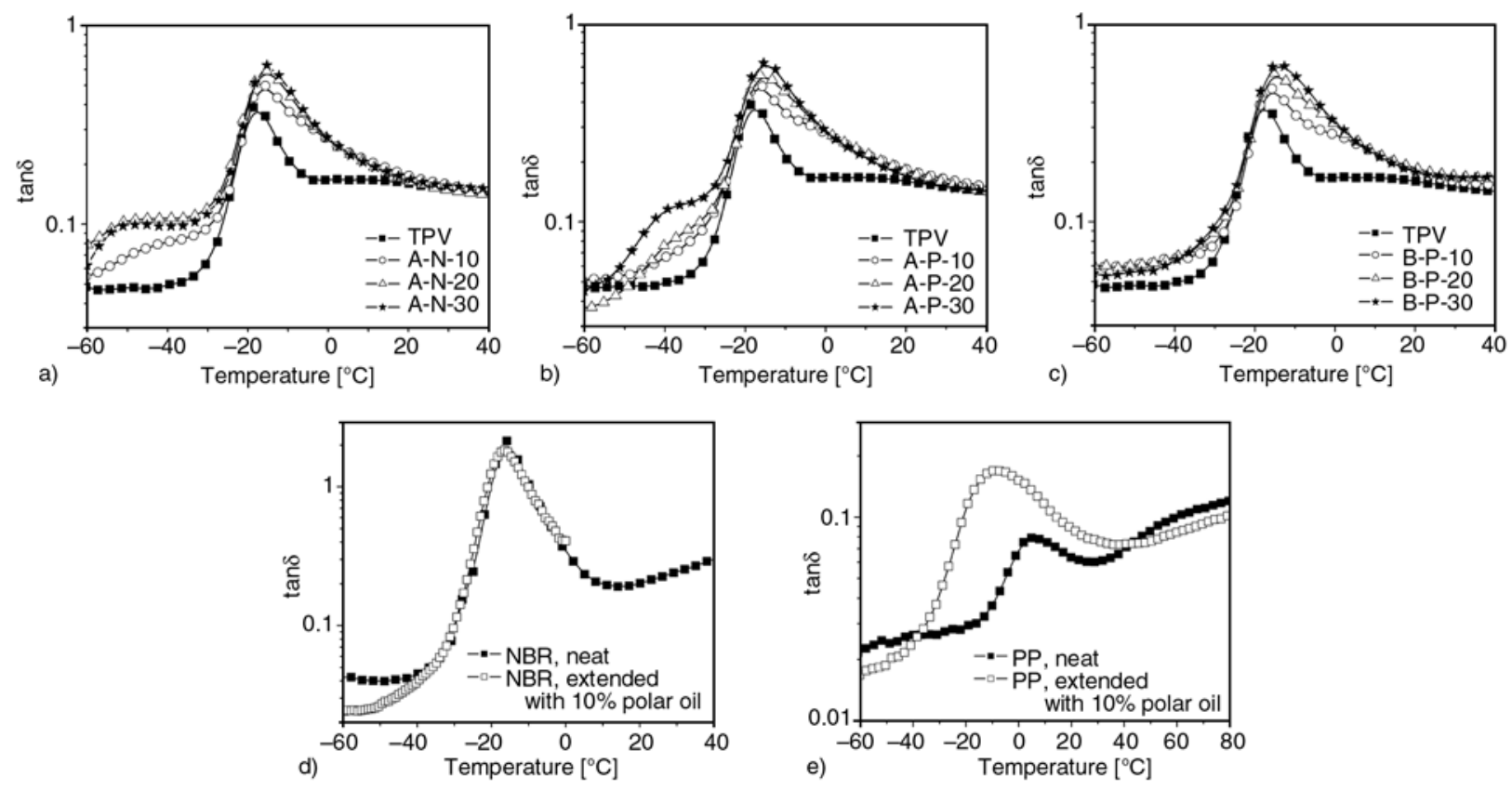

Figure 2. Temperature dependence of the loss factor tan $\delta$ for A-N (a), A-P (b), B-P (c), NBR (d) and PP (e)

contents added after the dynamic vulcanization process. Both in series A-N and A-P the size of the rubber domains is enlarged with increasing oil content. The added oil first accumulates in the PP matrix and then migrates into the rubber domains during the mixing process. It is worth to note that the NBR phase was cross-linked before oil addition, thus the cross-linking density of the NBR phase is not influenced by the oil immigration. At higher oil content of $30 \mathrm{wt} \%$ a bright phase is observed in the AFM micrographs which is related to a neat oil phase. This can be proved by taking into account the DMTA results. The temperature dependence of the loss factor $\tan \delta$ of the series A-N and A-P is presented in Figures $2 \mathrm{a}$ and $2 \mathrm{~b}$. The neat TPV shows the characteristic NBR $\alpha$-peak at $-18^{\circ} \mathrm{C}$ and a shoulder for the $\beta$-relaxation of PP at about $8^{\circ} \mathrm{C}$. Additionally, the oil extended TPVs show each a shoulder at lower temperatures of about -37 to $-50{ }^{\circ} \mathrm{C}$. These shoulders are related to the oil phase. The magnitude of the loss factor in this region increases with rising oil content. It is observed, that the peak of the rubber phase slightly shifts to higher temperatures with enhanced oil content. That can be explained as follows: The oil present in the rubber domains actually does not influence the position of the rubber peak as shown in Figure 2d, but the oil present in the PP matrix shifts the $\beta$-peak of PP toward lower temperatures (Figure 2e). This peak overlaps the peak of the rub- ber phase. As a result the rubber peak seems to shift towards higher temperatures.

When the oil is pre-mixed with rubber before the dynamic vulcanization process takes place, the rubber domains are comparatively large as seen in Figure 1c. The reason for this is the presence of oil in the rubber phase. The oil can hinder the vulcanization reaction and reduce the crosslink density [12] and thus the development of a fine dispersed rubber phase. At oil contents of 20 and $30 \mathrm{wt} \%$ a co-continuous phase morphology is observed, and no separate oil phase is visible in the AFM images (Figure 1c). Looking at the curve of the loss factor presented in Figure 2c, it is obvious that the peak of the oil is disappeared. Although the oil was premixed only in the rubber phase, it is interesting to see that there is an effect caused by the oil-modified PP phase. The relaxation peak of rubber in the system B-P shifts to higher temperatures due to the overlapping with the $\beta$-PP peak. It can be concluded, that the oil pre-mixed in the rubber phase diffused partly into the amorphous PP phase during the compounding process.

As shown in our previous work [4], the stress relaxation behavior is strongly determined by the crystalline phase of TPV. Using DSC the crystallization temperature $T_{c}$, melting temperature $T_{m}$, and degree of crystallinity $X_{c}$ of the PP phase in TPV were investigated and the results are summarized in Table 4. $T_{c}$ and $T_{m}$ of PP in TPV decrease with 
Table 4. Degree of crystallinity and thermal parameters of TPV investigated

\begin{tabular}{|c|c|c|c|}
\hline Sample name & $\mathbf{X}_{\mathbf{c}}[\mathbf{\%}]$ & $\mathbf{T}_{\mathbf{c}}\left[{ }^{\circ} \mathbf{C}\right]$ & $\mathbf{T}_{\mathbf{m}}\left[{ }^{\circ} \mathbf{C}\right]$ \\
\hline TPV & 17.8 & 124 & 163 \\
\hline A-N-10 & 15.9 & 117 & 158 \\
\hline A-N-20 & 13.4 & 114 & 155 \\
\hline A-N-30 & 12.8 & 112 & 152 \\
\hline A-P-10 & 14.9 & 117 & 157 \\
\hline A-P-20 & 14.1 & 113 & 155 \\
\hline A-P-30 & 13.2 & 110 & 152 \\
\hline B-P-10 & 16.6 & 117 & 161 \\
\hline B-P-20 & 15.0 & 108 & 158 \\
\hline B-P-30 & 14.6 & 104 & 157 \\
\hline
\end{tabular}

gradual incorporation of oil. Oil polarity, content and distribution in TPV affect the extent to which the $T_{c}$ and $T_{m}$ of PP drop down. Crystallinity is also diminished with the increasing amount of oil. The presence of oil and rubber domains affects the crystallinity of these vulcanizates by influencing the perfection of the growing iPP crystals. Similar findings were reported by other researchers [13].

\subsection{Short term mechanical behavior}

Figure 3a shows a comparison between the stressstrain behavior of TPV containing different types and contents of oil added after the dynamic vulcanization process. The presence of oil in the rubber and/or matrix phase significantly affects the mechanical properties [14]. The values of both stress and strain at break are reduced with increasing oil content as compared to neat TPV. Both A-N and A-P samples show a comparable behavior due to the presence of similar crystalline thermoplastic and cross-linked rubber phases.

For B-P samples in which oil was pre-mixed in the rubber phase the slope of the stress-strain curves in the range of larger deformations is strongly reduced. This feature is typical for the TPV with a low cross-linking density of the elastomer phase [15]. As discussed above, the decrease of the crosslinking density is caused by the presence of oil in the NBR phase (Figure 3b).

The residual strain as a measure for the elastic behavior of the TPVs investigated was characterized by hysteresis tests. As shown in Figure 4, a good strain recovery was observed for the A-N and A-P samples with increasing oil content due to the reduced crystallinity of the TPV. In contrast, the residual strain of B-P samples increases with rising oil content mainly due to the decreased crosslink density.
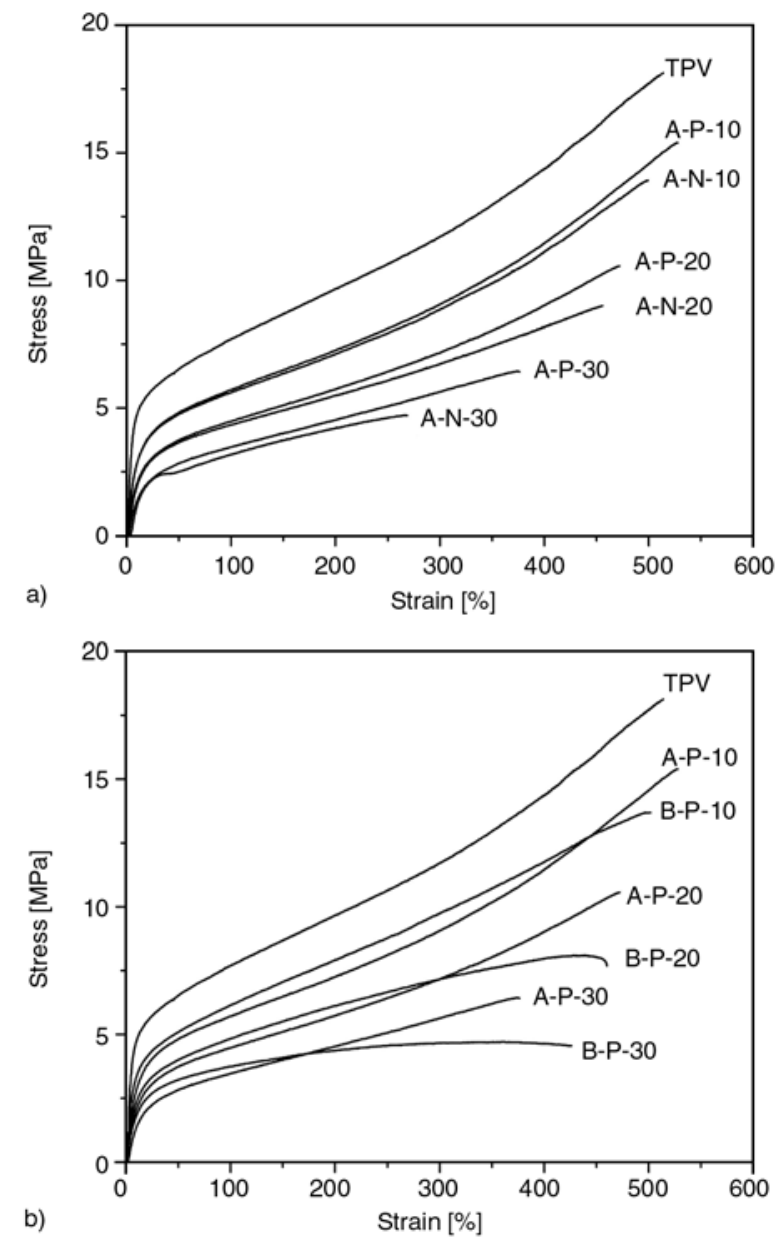

Figure 3. Stress-strain behavior of neat and oil extended TPV, A-P compared with A-N (a), A-P compared with B-P (b)

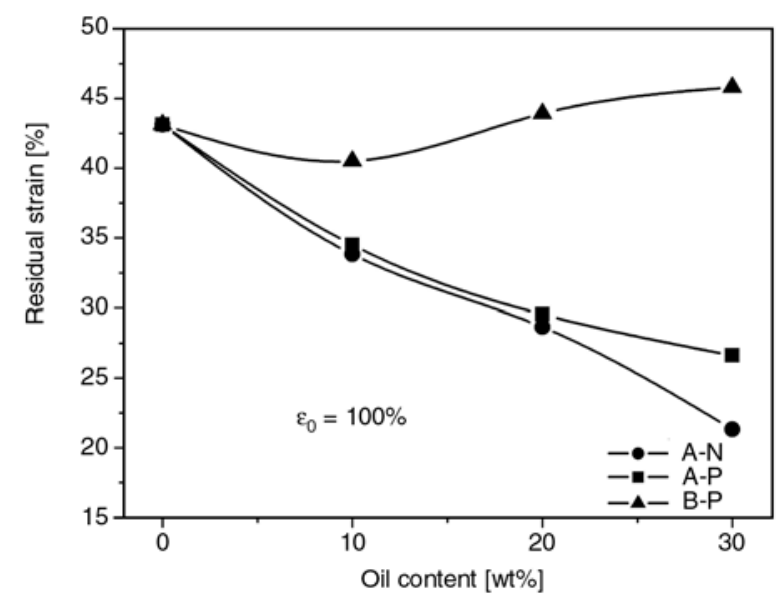

Figure 4. Residual strain measured from hysteresis experiments

\subsection{Stress relaxation behavior}

\subsubsection{Stress relaxation behavior of neat TPV}

In our previous work the stress relaxation behavior of neat TPV has already been studied using the 
two-component model [4]. To evaluate the relaxation curve, the applied stress $\sigma$ is separated into two components, a relaxing stress component $\Delta \sigma$ and a non-relaxing stress component $\sigma_{\infty}$ according to Equation (1) [8-10]:

$$
\sigma(t)=\Delta \sigma(t)+\sigma_{\infty}
$$

According to Seeger [8] the relaxing stress component $\Delta \sigma(t)$ is called thermal stress component, because it acts on short-range obstacles, which can be overcome by stress aided thermal activation. It depends on the plastic deformation rate $\dot{\varepsilon}$ and the temperature $T$ according to Eyring's rate theory [9]. In contrast to the relaxing stress component, the non-relaxing stress component $\sigma_{\infty}$ is called athermal stress component. $\sigma_{\infty}$ is originated from longrange stress fields, which cannot be overcome by thermal activation.

Figure 5a shows the stress relaxation behavior of neat TPV measured at different temperatures. With increasing temperature the relaxation curves shift horizontally to shorter times and vertically to lower
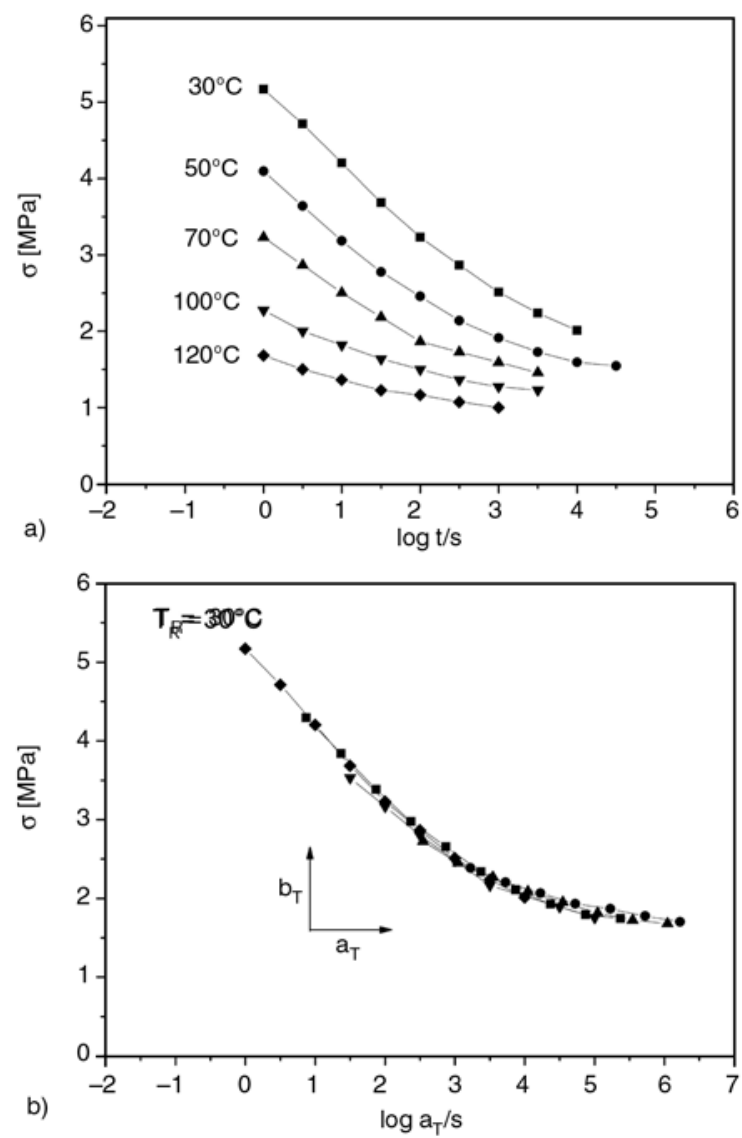

Figure 5. Stress relaxation curves of neat TPV measured at different temperatures (a) and master curve created by vertical and horizontal shift procedure (b) stress. The horizontal shift is caused by a reduction of the thermal stress component $\Delta \sigma$ and the vertical shift of the relaxation curves originates from a linear decrease of the athermal stress component $\sigma_{\infty}[16]$.

The procedure for creation of a master curve as described in our previous work [4] consists of a vertical and a horizontal shift of the curves to the reference curve measured at the reference temperature $T_{R}=30^{\circ} \mathrm{C}$. The vertical shift factor $b_{T}$ is determined by the temperature dependence of the athermal stress component as shown in Equation (2):

$b_{T}=\sigma_{\infty}\left(T_{R}\right)-\sigma_{\infty}(T)$

The master curve for a reference temperature of $30^{\circ} \mathrm{C}$ determined from the experimental results is shown in Figure $5 b$. A very good fit is observed for the experimental data.

In Figure 6 the temperature dependence of the horizontal and vertical shift factor of the neat TPV is presented. The temperature dependence of the horizontal shift factors $a_{T}$ follows the Arrhenius approach corresponding to Equation (3):

$\log a_{T}=A+\frac{0.43 E_{A}}{R T}$

The Arrhenius diagram (Figure 6a) shows a straight line at temperatures between 30 and $120^{\circ} \mathrm{C}$ that means that only one relaxation process takes place in the investigated temperature range. From the slope of the line an activation energy of $E_{A}=$ $90 \mathrm{~kJ} / \mathrm{mol}$ was calculated. This activation energy is close to the activation energy of the $\alpha$-relaxation process of the crystalline PP phase measured in this temperature range determined in our previous work $\left(E_{A}=110 \mathrm{~kJ} / \mathrm{mol}\right)[4,16]$ and to the corresponding results in the literature $(107-150 \mathrm{~kJ} / \mathrm{mol})$ [17].

In Figure $6 \mathrm{~b}$ the temperature dependence of the vertical shift factor $b_{T}$ determined by the athermal stress component is presented as a straight line. The larger the slope, the more strongly the relaxation curve shifts vertically to lower stresses at increasing temperature. It is obvious that the athermal stress component $\sigma_{\infty}$ consists of two contributions, namely, $\sigma_{\infty}^{P P}$ and $\sigma_{\infty}^{N B R}$. As discussed in $[4,16,18$, 19], the decrease of the athermal stress component $\sigma_{\infty}^{P P}$ of the PP phase with increasing temperature 

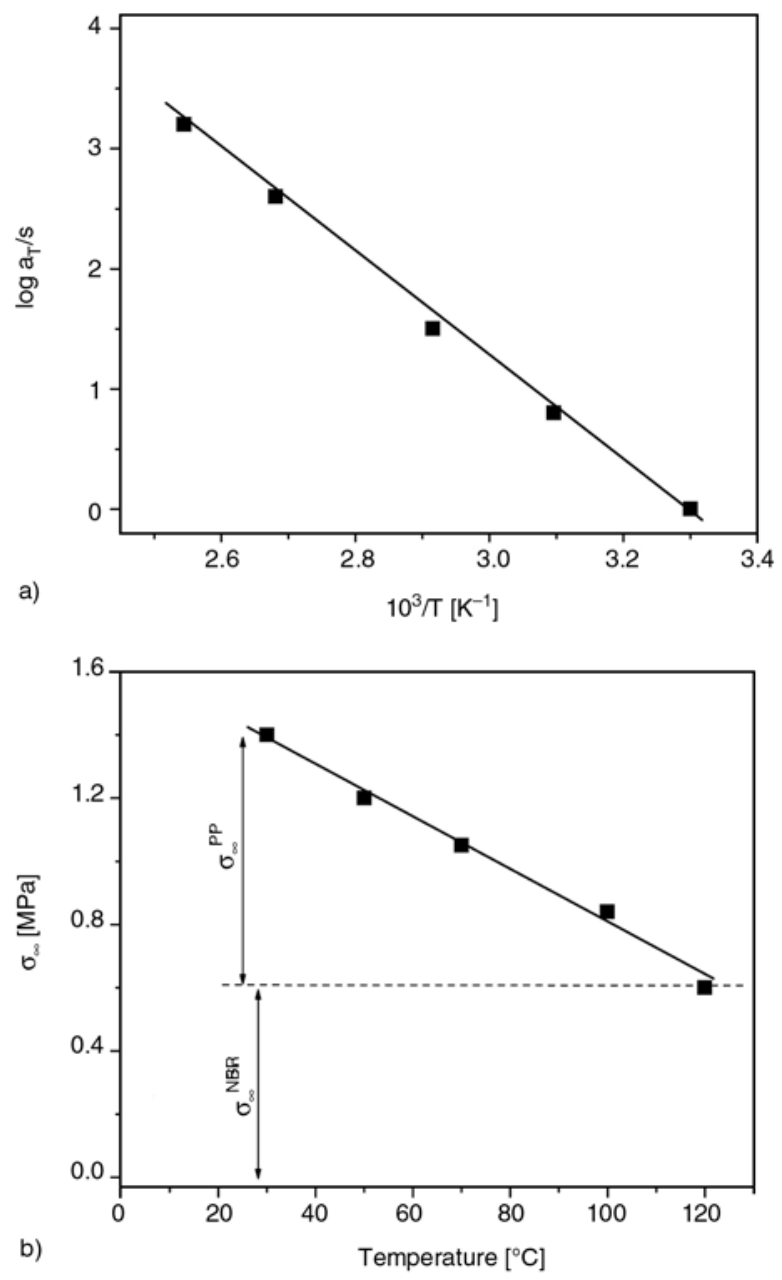

Figure 6. Temperature dependence of the horizontal shift factor $a_{T}$ (a) and athermal stress component $\sigma_{\infty}$ of neat TPV $(\mathrm{NBR} / \mathrm{PP}=65 / 35)(\mathrm{b})$

relates to the temperature dependent structural changes of the crystalline network of the PP phase. The shape of network points created by the PP lamella changes due to the slippage process of the PP lamella which is accelerated at higher temperatures. The athermal stress contribution of the crystalline PP phase $\sigma_{\infty}^{P P}$ decreases linearly and becomes zero at $120^{\circ} \mathrm{C}$. Thus, the value of the athermal stress component $\sigma_{\infty}$ at this temperature is determined only by the contribution of the rubber phase. The athermal stress component $\sigma_{\infty}^{N B R}$ of the NBR phase is caused by the deformation of the chemical network made by the cross-link points. It increases with increasing temperature according to the entropy elasticity theory described in Equation (4) [20]:

$\sigma_{\infty}^{N B R}=\left(\lambda-\lambda^{-2}\right) \cdot E_{\infty}^{N B R}=\left(\lambda-\lambda^{-2}\right) \cdot \frac{3 \rho k T}{M_{C}^{N B R}}$
In Equation (4) the rubber elastic modulus $E_{\infty}^{N B R}$ is calculated from the athermal stress component $\sigma_{\infty}^{N B R}$ and the draw ratio $\lambda$. It depends also on the density $\rho$, the Boltzmann constant $k$, the absolute temperature $T$ and the average molecular weight between the crosslinks $M_{C}^{N B R}$. In comparison to the big change of $\sigma_{\infty}$ the temperature dependent change of $\sigma_{\infty}^{N B R}$ can be considered as insignificant.

\subsubsection{Stress relaxation behavior of oil extended TPV}

The oil effect on the stress relaxation behavior of TPV can be discussed structurally by two quantities: the activation energy $E_{A}$ and the athermal stress component $\sigma_{\infty}$. They characterize two different temperature-dependent processes, i.e. the viscoelastic processes and the temperature dependent structural changes.

The temperature dependence of the horizontal shift factor $a_{T}$ of all oil extended TPV can be described only by one straight line as shown in Figure 7 . From the slope of the straight line an activation energy of $87 \mathrm{~kJ} / \mathrm{mol}$ was calculated, which displays the $\alpha$-relaxation process of the crystalline PP phase as the main viscoelastic deformation process taking place during the relaxation experiment and it does not depend on the oil content, its polarity and distribution in TPV.

The effect of oil addition on the athermal stress component for all systems is presented in Figure 8 at two test temperatures 30 and $120^{\circ} \mathrm{C}$. As discussed in our previous works $[4,16]$ at high temperatures, e.g. $120^{\circ} \mathrm{C}, \sigma_{\infty}^{P P}$ becomes zero, thus, only

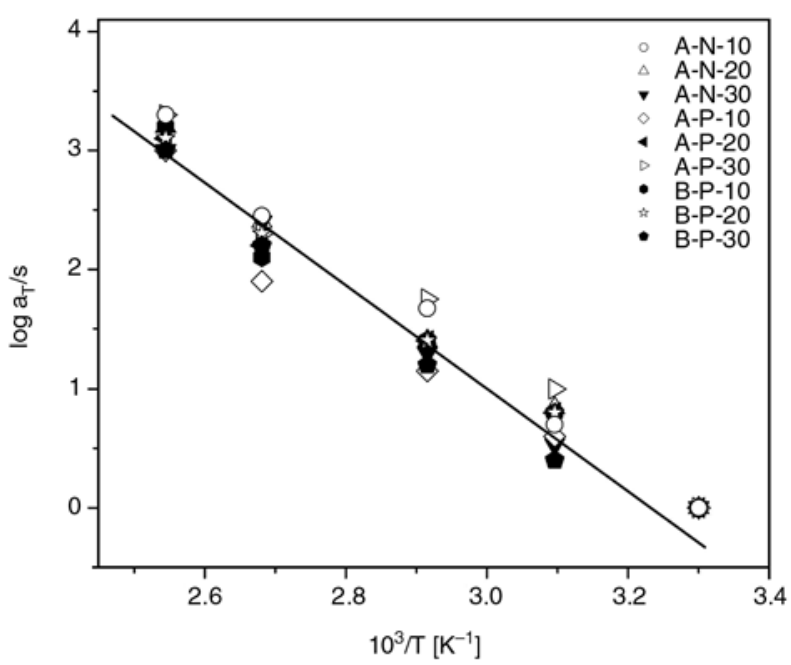

Figure 7. Temperature dependence of the horizontal shift factor $a_{T}$ of oil extended TPV 

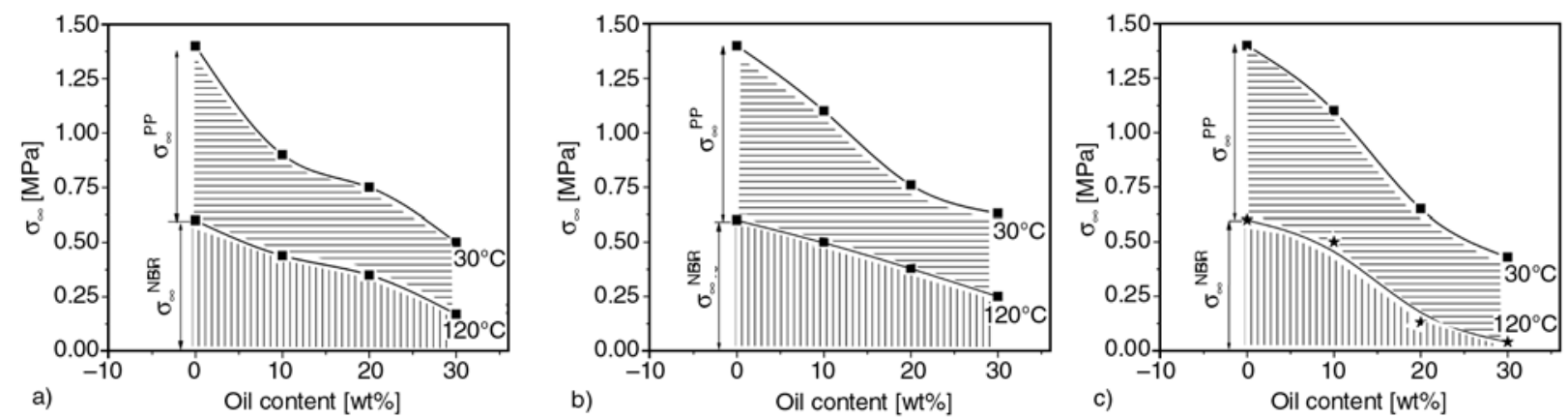

Figure 8. Effect of oil content on the athermal stress component $\sigma_{\infty}$ for A-N (a), A-P (b) and B-P (c) samples at different temperatures

the cross-linked rubber phase contributes to the athermal stress component. As a result, we can distinguish the contribution of the PP phase and NBR phase in dependence on the oil content as shown in Figures $8 \mathrm{a}, 8 \mathrm{~b}$ and $8 \mathrm{c}$ for three systems. It is obvious that in all systems both athermal stress components $\sigma_{\infty}^{P P}$ and $\sigma_{\infty}^{N B R}$ decrease differently with the oil addition. Because the athermal stress component is directly determined by the cross-link state, an attempt has been done in order to determine the average molecular weight between two crosslinks $M_{C}^{P P}$ and $M_{C}^{N B R}$ for both phases according to Equation (4). The calculated $M_{C}^{P P}$ and $M_{C}^{N B R}$ in dependence on the oil content for three systems are presented in Figure 9.

For the PP phase, the average molecular weight between two crosslinks (lamellae) $M_{C}^{P P}$ of the three systems increased with increasing oil content. Due to the oil immigration process in all systems oil was always present in the PP phase regardless of the different mixing regime. Oil existent in PP swells the PP phase to a larger volume on the one hand, and

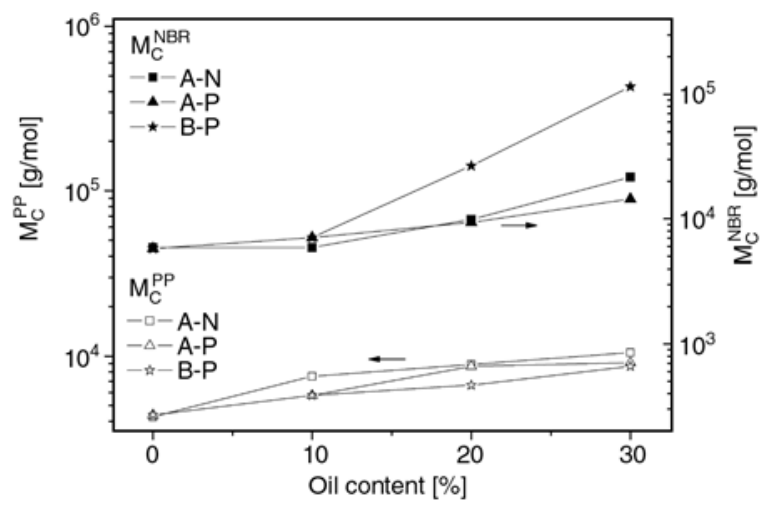

Figure 9. Average molecular weight between two crosslinks $M_{C}^{P P}$ and $M_{C}^{N B R}$ of the PP and NBR phase, respectively, in dependence on the oil content on the other hand oil causes a slight decrease of the crystallinity $X_{C}$ (Table 4). The decrease of $X_{C}$ leads to a reduction of crystalline cross-links (lamellae) in the PP phase that also contributes to the increase of $M_{C}^{P P}$. Only a minor difference between $M_{C}^{P P}$ of the three systems is observed. That means the quantity of oil in the PP phase seems to be the same.

In the systems A-N and A-P the oil addition does not affect the dynamic vulcanization process and thus the average molecular weight between two chemical cross-links $M_{C}^{N B R}$ of the NBR phase. However, the calculated $M_{C}^{N B R}$ of the three systems increases with increasing oil content in different manner (Figure 9). Thus is worth to note that the calculated $M_{C}^{N B R}$ is not a true value but only an apparent one. That is related to the fact that the oil is homogenously and finely dispersed in the rubber domains and thus the oil extended rubber domains are considered as a uniform phase having a larger volume compared to the non-extended rubber phase. For an oil extended rubber compound a true $M_{C}$ can be determined by use of the extraction experiment when oil is extracted from the compound [21]. Using Equation (4) only an apparent $M_{C}$ is calculated and it often differs from the true value [22]. In Figure 9 the similar increase of $M_{C}^{N B R}$ in these systems is related to the swelling effect of the oil present in the NBR phase. A strong increase of $M_{C}^{N B R}$ in the system B-P was observed at an oil content of more than $10 \%$. Beside the swelling effect of the added oil the ineffective cross-link reaction of the NBR phase in presence of the oil is the main reason for the high value of $M_{C}^{N B R}$. That leads accordingly to the diminishment of the athermal stress component $\sigma_{\infty}^{N B R}$ in the system B-P (Figure $8 \mathrm{c}$ ). 


\section{Conclusions}

In the present work the stress relaxation behavior of the oil extended thermoplastic vulcanizates (TPV) has been characterized by means of the two-component model which allows splitting the initial stress into two components: a thermal activated stress component and an athermal one. It was found that the oil distribution strongly affects the athermal stress component which is related to the contribution of the structural changes, e.g. crystallinity of the PP phase and the average molecular weight between two cross-links of the NBR phase. A master curve was created by shifting the relaxation curves vertically and horizontally towards the reference curve. The vertical shift factor $b_{T}$ is determined from the temperature dependence of the athermal stress component. From the temperature dependence of the horizontal shift factor $a_{T}$ the main viscoelastic relaxation process was determined as the $\alpha$-relaxation process of the crystalline PP phase, which is not dependent on the polarity and content of the oil as well as the mixing regime.

\section{Acknowledgements}

The authors gratefully acknowledge the financial support by Deutsche Forschungsgemeinschaft (DFG).

\section{References}

[1] Coran A. Y., Patel R. P.: Rubber-thermoplastic compositions. Part I. EPDM-polypropylene thermoplastic vulcanizates. Rubber Chemistry and Technology, 5, 141-150 (1980).

[2] Xiao H. W., Huang S. Q., Jiang T.: Morphology, rheology, and mechanical properties of dynamically cured EPDM/PP blend: Effect of curing agent dose variation. Journal of applied Polymer Science, 92, 357-362 (2004).

[3] Babu R. R., Singha N. K., Naskar K.: Studies on the influence of structurally different peroxides in polypropylene/ethylene alpha olefin thermoplastic vulcanizates (TPVs). Express Polymer Letters, 2, 226-236 (2008).

[4] Le H. H., Lüpke T., Pham T., Radusch H-J.: Time dependent deformation behavior of thermoplastic elastomers. Polymer, 40, 4589-4597 (2003).

[5] Long D., Sotta P.: Stress relaxation of large amplitudes and long timescales in soft thermoplastic and filled elastomers. Rheologica Acta, 46, 1029-1044 (2007).
[6] Baeurle S. A., Hotta A., Gusev A. A.: A new semiphenomenological approach to predict the stress relaxation behavior of thermoplastic elastomers. Polymer, 46, 4344-4354 (2005).

[7] Asaletha R., Bindu P., Aravind I., Meera A. P., Valsaraj S. V., Yang W., Thomas S.: Stress-relaxation behavior of natural rubber/polystyrene and natural rubber/polystyrene/natural rubber-graft-polystyrene blends. Journal of Applied Polymer Science, 198, 904-913 (2008).

[8] Seeger A.: Handbuch der Physik. Springer, Berlin (1958).

[9] Krausz A. S., Eyring H.: Deformation kinetics. Wiley, New York (1975).

[10] Ferry J. D.: Viscoelastic Properties of polymers. Wiley, New York (1980).

[11] Li J. C. M.: Dislocation dynamics in deformation and recovery. Canadian Journal of Physics, 45, 493-509 (1967).

[12] Le H. H., Prodanova I., Ilisch S., Radusch H-J.: Online electrical conductivity as a measure to characterize the carbon black dispersion in oil containing rubber compounds with a different polarity of rubber. Rubber Chemistry and Technology, 77, 815-829 (2004).

[13] Yang Y., Chiba T., Saito H., Inoue T.: Physical characterization of a polyolefinic thermoplastic elastomer. Polymer, 39, 3365-3372 (1998).

[14] Baranov A. O., Erina N. A., Medintseva T. I., Kuptsov S. A., Prut E. V.: The influence of the interphase layer on the properties of isotactic polypropylene/ethylenepropylene rubber blends. Polymer Science, Series A, 43, 1177-1183 (2001).

[15] Huy T. A.: Rheo-optische Charakterisierung des Deformationsverhaltens dynamischer Vulkanisate. PhD Thesis, Martin Luther University Halle-Wittenberg, Germany (1999).

[16] Le H. H.: Spannungsrelaxationsuntersuchungen zur Charakteiserung des zeitabhängigen Deformationsverhaltens thermoplastischer Elastomere. PhD Thesis, Martin Luther University Halle-Wittenberg, Germany (2002).

[17] Brandrup J., Immergut E. H.: Polymer handbook. Wiley, New York (1989).

[18] Shapery R. A.: On the characterization of nonlinear viscoelastic materials. Polymer Engineering and Science, 9, 295-310 (1969).

[19] Boyd R.: Relaxation processes in crystalline polymers: Experimental behaviour -A review. Polymer, 26, 323-347 (1985).

[20] Treloar L. R. G.: The physics of rubber elasticity. Clarendon Press, Oxford (1975).

[21] Flory P. J.: Principles of polymer chemistry. Cornell University Press, Ithaca, New York (1953).

[22] Ilisch S., Menge H., Radusch H-J.: Vernetzungsausbeute an konventionellen und dynamischen Vulkanisaten. Kautschuk und Gummi Kunststoffe, 53, 206-212 (2000). 